\title{
GLAD!
}

Revue sur le langage, le genre, les sexualités

11 | 2021

Archives, genre, sexualités, discours

\section{Féminisme radical et sexualité : « Le manifeste de l'asexualité » de Lisa Orlando (1972)}

Radical Feminism \& Sexuality: The Asexual Manifesto by Lisa Orlando (1972)

\section{Élie Grau et Lisa Orlando}

\section{(2) OpenEdition}

Édition électronique

URL : https://journals.openedition.org/glad/3452

DOI : $10.4000 /$ glad.3452

ISSN : 2551-0819

Éditeur

Association GSL

\section{Référence électronique}

Élie Grau et Lisa Orlando, « Féminisme radical et sexualité : « Le manifeste de l'asexualité » de Lisa Orlando (1972) », GLAD! [En ligne], 11 | 2021, mis en ligne le 20 décembre 2021, consulté le 08 décembre 2022. URL : http://journals.openedition.org/glad/3452 ; DOI : https://doi.org/10.4000/glad. 3452

Ce document a été généré automatiquement le 29 janvier 2022.

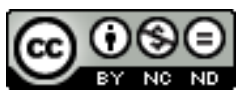

Creative Commons - Attribution - Pas d'Utilisation Commerciale - Pas de Modification 4.0 International - CC BY-NC-ND 4.0

https://creativecommons.org/licenses/by-nc-nd/4.0/ 


\title{
Féminisme radical et sexualité : « Le manifeste de l'asexualité » de Lisa Orlando (1972)
}

\author{
Radical Feminism \& Sexuality: The Asexual Manifesto by Lisa Orlando (1972)
}

\author{
Élie Grau et Lisa Orlando
}

1 En juin 2019, un document de 1974 retrouvé assez fortuitement a fait le tour des communautés asexuelles en ligne. "Enfin une source importante pour l'histoire des asexuelles avant les années 2000 !»a-t-on pensé. Mais la réalité est peut-être plus complexe. En lisant l'appel à articles de GLAD!, j'avais aussitôt pensé à ce document qui a eu, en quelque sorte, deux vies: l'une dans les années 1970 et l'autre, désormais virtuelle, depuis 2018 (date à laquelle l'existence du manifeste a été redécouverte sans qu'on soit encore arrivé à le localiser).

Ce «Manifeste de l'asexualité » a été écrit en 1972 au sein d'un groupe de réflexions féministes, les New York Radical Feminists [Féministes Radicales de New York]. Ce groupe avait été fondé en 1969 par une ancien-ne membre du groupe The Feminists [Les Féministes] qui l'avait quitté suite à une divergence d'opinion. Suite à ce groupe de réflexion, à l'écriture de ce manifeste et à son succès, Lisa Orlando s'est vue d'ailleurs approchée par The Feminists pour les rejoindre (ce qu'elle a refusé de faire). ${ }^{1}$

3 En 1972, donc, les membres des NYRF décidèrent de créer deux comités (caucus) pour explorer le problème de la division des féministes entre les hétérosexuelles et les lesbiennes. Un troisième comité fut créé pour les bisexuelles puis un quatrième, pour les « asexuelles » que Orlando décrivit comme « les ex-lesbiennes refroidies après avoir découvert que les femmes ne sont pas meilleures que les hommes ». Une seule femme Barbara Getz - se joignit à ce comité. Les comités devaient rendre un rapport, ce que seul le comité d'Orlando fit. Le manifeste actuel est une réécriture de ce rapport, plus proche des convictions personnelles d'Orlando, Getz refusant désormais d'y être associée. 
4 Pour autant, le manifeste, publié et distribué par les NYRF, eut son petit succès, semblet-il : Evan Morley écrivit dans la newsletter des NYRF à quel point iels recevaient des demandes pour le document ; un extrait se trouve aussi dans Sexual Honesty, un livre de 1974, écrit par Shere Hite ; le manifeste fut lu et discuté en direct sur la station de radio WBAI pendant une des émissions l'Heure du Loup (Hour of the Wolf) de Margot Adler. Le manifeste fut aussi cité dans le journal The Gay Liberator $n^{\circ} 48$ (printemps 1976). L'auteur de l'article est Greg Turner, qualifié de « libérationiste gay » et originaire de Détroit. En décembre 1972, Barbara Getz anima un atelier sur l'asexualité lors du « Dialogue entre Lesbiennes et Féministes » organisé au Barnard College, à New York. Un compte-rendu de cette journée fut publié dans le numéro de janvier 1973 du journal off Our Backs et une note renseigne les lecteurices sur la façon de se procurer le manifeste.

5 La copie du manifeste qui se trouve aujourd'hui sur Internet vient des archives de l'ALFA, Atlanta Lesbian Feminist Alliance [Alliance des Féministes Lesbiennes d'Atlanta]. L'ALFA fut fondée en 1972 et perdura jusqu'en 1994. Outre le fait qu'elle est considérée comme l'une des plus vieilles organisations de féministes lesbiennes des États-Unis, l'Alliance s'est préoccupée de la question des archives dès son commencement. Ces archives comprenaient ainsi les registres de l'ALFA, mais aussi ceux d'autres groupes féministes du sud, une énorme collection de périodiques féministes, lesbiens ou militants et une bibliothèque de livres féministes ou gays/ lesbiens.

6 Mais l'histoire du manifeste s'interrompt bien vite ; les NYRF cessent d'exister, semblet-il, à la fin des années 1980, tout comme beaucoup de ces autres groupes féministes ou gay qui ne comptaient d'ailleurs jamais un grand nombre de membres et se scindaient régulièrement. En réalité, ce manifeste est tout autant une source pour l'histoire de l'asexualité qu'un témoin du milieu féministe militant des années 1970 aux États-Unis : un milieu foisonnant d'idées politiques où les écrits s'échangeaient de mains en mains ou se discutaient de vive voix entre les mêmes personnes qui se retrouvaient à chaque évènement.

7 Pour bien comprendre les propos du manifeste, il ne faut pas oublier le contexte de l'époque : la révolution sexuelle. Ela Przybylo en rend compte dans son livre, Asexuals Erotics : faire montre de signes d'un désir sexuel en public devient une nécessité sociale. Un livre de 1962 condamne les femmes frigides. Pour certaines féministes, cela a conduit au rejet de l'acte sexuel lui-même : Valerie Solanas, dans le SCUM Manifesto de 1967, considère l'acte sexuel comme une invention du patriarcat - qui serait dès lors à rejeter. (Il est intéressant de noter que Ti-Grace Atkinson qui soutint Solanas est l'une des fondatrices du groupe The Feminists.) Dans Independence from the Sexual Revolution, Dana Densmore écrit en 1971: «Le droit qui est un devoir. La liberté sexuelle qui n'inclut pas la liberté de refuser l'acte sexuel, de refuser d'être défini à chaque instant par le sexe. L'acte sexuel devient une religion. »

8 L'asexualité décrite par Lisa Orlando dans ce manifeste n'est donc pas la description d'une orientation sexuelle, mais bien un choix découlant d'une réflexion féministe. En réalité, cette asexualité-là est à rapprocher du lesbianisme politique qui pouvait prendre la forme d'un refus des femmes hétérosexuelles de nouer des relations romantiques/sexuelles avec les hommes sans pour autant en nouer avec des femmes. Malgré tout, une asexuel-le lisant ce manifeste y reconnaitra des problématiques personnelles familières comme la remise en question de l'acte sexuel comme base et ciment de toute bonne relation romantique. 
9 Mais que faire de ce manifeste aujourd'hui ? Je le disais en préambule : la redécouverte de ce document a suscité l'extase dans les sphères asexuelles. L'histoire des asexuellles est compliquée à écrire car invisible; les mots pour décrire cette orientation sexuelle ont manqué pendant longtemps et pendant tout aussi longtemps, les asexuelles ont pu être groupées avec les bisexuelles. C'est pour cette raison que la communauté "ace " ne s'est réellement épanouie que sur Internet, via $\mathrm{AVEN}^{2}$ ou sur des blogs hébergés sur Tumblr. Cet état de fait a pu lui valoir la réputation de n'être qu'un produit d'Internet, une mode des années 2000. C'est pour cette raison que le manifeste d'Orlando est apparu comme si important, non pas pour ce qu'il raconte, mais à cause de sa date et sa mention explicite d'« asexualité ». Cependant, en faire une étape dans l'histoire de la reconnaissance de l'asexualité, c'est ignorer le milieu qui l'a produit et son objectif premier qui était non pas de définir un aspect méconnu de la sexualité humaine, mais de réfléchir sur les enjeux des relations humaines elles-mêmes.

10 Il est à espérer qu'à mesure que l'histoire de l'asexualité - et des communautés LGBTQIA+ en général - sera étudiée, nous puissions nous repencher sur ce genre de documents et réouvrir des sujets de discussion oubliés dans de vieilles boites d'archives, comme celles de l'AFLA.

Élie Grau

\section{Manifeste de l'Asexualité}

11 En septembre 1972, le Conseil coordonnant des Féministes Radicales de New York (Coordinating Council of New York Radical Feminists) créa des comités (caucus) sur la base des similarités entre les orientations sexuelles. Chaque comité devait explorer les comportements personnels et politiques de ses membres en rapport avec leur sexualité et communiquer ces points de vue au groupe entier. Barbie Hunter Getz et moi-même [Lisa Orlando] avons pris conscience que nous ne nous serions senties à l'aise dans aucun des comités proposés (hétérosexuel, lesbien, bisexuel) et avons créé le nôtre. De ce comité est sorti un article dont le "Manifeste de l'asexualité » est une révision. L'usage de la première personne du pluriel retenu dans cet article n'implique pas que toutes les opinions exprimées dans cette version finale reflètent nécessairement les opinions des deux co-autrices d'origine.

\section{Origine et définition}

12 Nos expériences de la sexualité n'ont pas été congruentes avec nos valeurs féministes. À mesure que notre conscience s'éveillait à cette problématique, nous avons commencé à voir à quel point le sexe imprégnait nos vies et celle des autres. Nous catégorisions nos relations en termes de sexe: soit amiee soit amante. Nous nous engagions dans un processus de comparaison à chaque nouvelle rencontre et, aussi subtil ou subconscient qu'il soit, ce processus fait que nous acceptions ou rejetions cette nouvelle personne comme potentielle partenaire sexuellle quand bien même nous n'avions aucun désir de nous impliquer sexuellement. Nous avons arbitrairement rejeté des groupes entiers de personnes en les classant comme inadéquates pour une relation intime parce que nous avons considéré que de telles relations, par définition, devaient nécessairement inclure l'acte sexuel. Le plus souvent, nous choisissons de passer du temps avec certaines personnes uniquement sur la base de leur disponibilité sexuelle (la « soirée au bar »3). À 
mesure que nous prenons conscience de ce fait vis-à-vis de nous-mêmes, nous prenons douloureusement conscience que nous avons été objectifiées par les autres de la même façon.

L'asexualité est un prolongement de cette prise de conscience. C'est un concept que nous en sommes venues à utiliser suite au désir de communiquer - non pas seulement à travers notre existence, mais aussi à travers notre langage - notre combat pour nous débarrasser du sexisme dans nos vies personnelles.

Dans cet article, nous avons utilisé les termes « sexe ", " acte sexuel » et " sexuel » pour décrire toute activité dont l'un des objectifs est l'excitation génitale ou l'orgasme. Les marques d'affection, physiques et sensuelles (y compris les baisers), ne sont pas, dans cette définition, sexuelles, à moins qu'elles ne soient réalisées en vue de l'excitation génitale.

avons choisi le terme d'« asexuelles » pour nous décrire car autant « célibataires » que " anti-sexuelles » avaient des connotations que nous souhaitons éviter: le premier sous-entend que l'on aurait renoncé à la sexualité pour un bien supérieur, le second que la sexualité serait dégradante ou mauvaise par nature. "Asexuelle», selon notre usage, ne veut pas dire "sans actes sexuels", mais "qui ne se lie sexuellement à personne ». Cela n'exclut bien évidemment pas la masturbation, mais sous-entend que si l'on a des élans sexuels, ils n'ont pas besoin d'une autre personne pour s'exprimer. L'asexualité est, simplement, une sexualité autonome.

\section{Philosophie}

16 Notre philosophie de l'asexualité a germé de nos valeurs morales personnelles et a été refaçonnée par notre conscience féministe. Pour nous, ainsi que pour tant d'autres femmes, le féminisme signifie davantage que la lutte contre le sexisme. Il signifie la sororité - une nouvelle façon de se lier aux autres, peut-être une nouvelle façon de vivre. La morale féministe, à ce point de l'Histoire, ne peut être définie que de façon antithétique par rapport aux valeurs oppressives de notre société (par exemple, la compétition, l'objectification). Au niveau personnel, il se reflète dans notre conviction que nous devrions essayer de nous lier aux autres dans leur totalité, autant que possible, et ne pas les voir comme des objets qui existeraient pour la satisfaction de nos besoins; que nous ne devons pas exploiter les autres - c'est-à-dire, les utiliser « de façon injuste ou inappropriée » - et ne devons pas non plus nous laisser exploiter ; que nous ne devons pas être malhonnêtes envers nous-mêmes ou envers celleux que nous respectons. Par ailleurs, nous croyons que nous avons chacune la responsabilité d'examiner notre comportement, de déterminer dans quelle mesure il a été affecté par un conditionnement sexiste, et de le changer s'il n'est pas à la mesure de nos attentes.

En tant que féministes, nous avons dénoncé l'exploitation sexuelle des femmes par les hommes sans voir que nous aussi, nous avons utilisé les autres « de façon injuste et inappropriée ». Le sexe interpersonnel n'est pas un mode de comportement instinctif ; c'est un comportement que nous avons appris à utiliser pour la satisfaction d'un besoin (pour l'orgasme) que nous pouvons facilement satisfaire par nous-mêmes. Nous en sommes venues à considérer cette utilisation d'autrui comme une exploitation et nous avons compris qu'en permettant aux autres de nous utiliser de la même façon, nous donnons notre accord à notre propre exploitation. 
Dans notre tentative d'être honnêtes avec nous-mêmes, nous avons essayé de déterminer quels sont nos véritables besoins. Nous avons vu que nous avons un besoin d'affection, de chaleur, de contact tactile, besoin qu'on nous a appris à satisfaire par le sexe interpersonnel. À mesure que nous avons commencé à satisfaire ces besoins au sein de nos "amitiés", notre besoin et notre intérêt dans l'acte sexuel ont diminué. Nous nous sommes aussi rendu compte que nous avons un besoin d'intimité, un état que nous avons toujours considéré comme " atteint " au travers de l'acte sexuel. Avec le recul, nous avons compris que nous, comme les autres, avions utilisé l'acte sexuel comme un moyen de nous aveugler nous-mêmes, d'éviter la véritable intimité plutôt que d'y aboutir.

Nous avions combattu notre propre conditionnement de plein de façons différentes, surtout en termes de rôles, mais nous avions évité d'examiner la base du conditionnement qui a façonné notre sexualité. Il est difficile de spéculer sur la nature même d'une "sexualité idéale " (non influencée par le sexisme), mais nous sommes convaincues qu'elle n'occuperait pas autant de place dans nos vies que ce qui est actuellement le cas dans notre société. Nous vivons dans une culture d'« adorateurices de fétiches", qui considèrent l'acte sexuel avec une attention aussi extrême qu'irrationnelle. De même que beaucoup d'entre nous ont été conditionnées pour consacrer leur énergie à la préparation de somptueux repas, créant un fétiche par notre simple besoin de ne pas avoir à affronter le vide de nos vies de femmes, de même avons-nous été conditionnées pour rechercher la satisfaction sexuelle par des voies détournées et alambiquées. Depuis le début de notre implication dans le féminisme, nos vies ont acquis tellement plus de sens que nous n'avons plus besoin de fétiches.

Après examen de nos expériences selon nos valeurs, nous sommes parvenues à une asexualité qui est à la fois une revendication et une manière d'être. Le sexe interpersonnel ne nous est plus important, plus suffisamment pour justifier le rôle destructeur et perverti qu'il a joué dans nos relations. Il ne définit plus nos relations et en aucun cas ne constitue nos identités. En tant que femmes asexuelles, (1) nous ne recherchons, commençons ou n'entretenons aucune relation dans le but de faire l'expérience du sexe interpersonnel, (2) nous n'utilisons pas les autres pour la satisfaction de nos besoins sexuels et ne permettons pas que nous soyons utilisées nous-mêmes, (3) nous n'essayons pas de satisfaire nos autres besoins (d'affection, de chaleur, d'intimité, par exemple) par l'usage du sexe interpersonnel, ou (4) nous ne percevons pas les autres selon leur potentiel, ou non-potentiel, de devenir des partenaires sexuelles. En substance, notre asexualité renvoie au rejet du sexe interpersonnel tant que celui-ci ne correspond pas à nos conditions : qu'il soit à la fois congruent avec nos valeurs, et complètement accessoire et sans aucune importance pour nos relations.

\section{Politique}

21 À la base de la libération des femmes se trouve la destruction du sexisme, dont l'une des manifestations est l'exploitation sexuelle des femmes par les hommes. L'asexualité est une étape de plus pour atteindre notre objectif au niveau personnel, considérant qu'elle élimine une des façons que les hommes ont de nous opprimer. À travers notre asexualité, nous avons exclu l'acte sexuel comme objectif et même, fondamentalement, comme possibilité dans toute relation que nous pourrions nouer avec des hommes. 

comportements oppressifs - normaux au sein d'une telle culture - ont rendu extrêmement difficile la possibilité pour les femmes de développer un style d'attachement aux autres qui leur soit propre, indépendant et plus humain. Par conséquent, la plupart des femmes reproduisent, dans leurs relations les unes avec les autres, certaines formes de ces comportements d'exploitation, caractéristiques de nos oppresseurs masculins. Un endroit où l'oppression des femmes par les femmes peut arriver est, une fois encore, dans le domaine du sexuel; cette oppression-là doit disparaitre, elle aussi, si nous voulons vraiment être libres. À travers l'asexualité, nous avons rejeté le sexe comme objectif dans nos relations avec les femmes, nous permettant ainsi d'éviter l'objectification sexuelle, l'exploitation et l'oppression de nos sœurs. Ici aussi, nous rejetons toute possibilité d'acte sexuel, sauf s'il remplit nos conditions, et nous nous gardons donc d'être sexuellement exploitées et opprimées.

Afin de détruire les mythes primaires d'une culture en particulier, il faut saper ses propres fondations. La culture patriarcale, qui repose sur la différenciation sexuelle, a construit certains de ses mythes les plus forts autour de la sexualité. Nous croyons qu'il est crucial que le féminisme se tourne vers la mise en lumière et la destruction de la mythologie patriarcale actuelle qui, par la tromperie, renforce notre oppression. Les principaux mythes responsables de la distorsion du rôle que le sexe joue dans la vie des femmes sont les suivants :

1. Le sexe interpersonnel est vital puisque la libido est un puissant moteur de la vie humaine et, si elle demeure insatisfaite (à travers le sexe interpersonnel), cela tend à provoquer un mal-être voire une maladie.

2. Il est important que toute excitation sexuelle soit toujours et/ou immédiatement satisfaite.

3. Le sexe est essentiel pour fortifier une relation (amoureuse), aucune relation n'est complète sans lui.

4. L'intimité ultime dans une relation se produit durant l'acte sexuel et/ou l'orgasme.

5. Le besoin d'affection physique ou de sexe c'est fondamentalement la même chose.

6. Il est presque impossible d'exprimer de l'affection physique d'une manière satisfaisante, sans que cela n'entraine une excitation sexuelle.

7. Les femmes qui ont peu d'intérêt pour le sexe interpersonnel ou qui n'atteignent que rarement (si ce n'est jamais) l'orgasme sont d'une certaine manière inadéquates (mal baisées, frigides).

S'il est vrai que toutes les femmes ne croient pas en tous ces mythes, certaines femmes croient à certains d'entre eux, en certaines circonstances.

En dernier point, nous voyons un conflit d'intérêts entre, d'un côté, le temps et l'énergie nécessaires à notre combat en tant que féministes et, de l'autre, le temps et l'énergie nécessaires pour développer et entretenir des relations qui ont pour objectif l'acte sexuel. Pour utiliser notre énergie avec efficacité, un choix semble devoir être fait : se battre contre le sexisme ou se battre pour du sexe satisfaisant. Il est certes vrai que tourner le dos à un problème ne le résout pas, mais nous pensons que la vérité de cette maxime est proportionnelle à l'importance que l'on donne au problème. Si nous avions considéré le sexe interpersonnel comme important, l'asexualité serait une façon d'esquiver un problème; puisque ce n'est pas le cas, c'est bien un moyen de retirer notre énergie d'une activité dans laquelle il nous semble que nous la gâchons en vain. 
Nous voyons l'asexualité comme un «style de vie alternatif » efficace pour les femmes révolutionnaires, mais nous ne revendiquons pas que l'asexualité soit la révolution. Nous nous qualifions de "femmes auto-identifiées", mais nous ne demandons pas que toutes les féministes adoptent ce titre. Notre déclaration est aussi simple que cela: suite à l'examen de la nature de notre sexualité et suite à la revendication d'une sexualité sans fausses idées sexistes, nous sommes capables de former et d'entretenir des relations d'une façon qui tout à la fois s'accorde à nos valeurs et est efficace dans notre combat pour notre libération. Pour nous, l'asexualité est un engagement à défier et en fin de compte à détruire les allégations sans fondement autour de l'acte sexuel et des relations, allégations qui soutiennent et perpétuent le patriarcat.

Lisa Orlando, Comité Asexuel, NYRF

\section{BIBLIOGRAPHIE}

DENSMORE, Dana. 1973. «Independence from the Sexual Revolution », in Radical Feminism, Koedt, Anne, Levine, Ellen \& Rapone, Anita. New York : Quadrangle, 107-118.

PRZYBYLO, Ela. 2019. Asexual Erotics. Columbus : Ohio State University Press.

SIGGY. 2019. «Lisa Orlando, Author of The Asexual Manifesto (1972)» The Asexual Agenda, consulté le 10 mai 2021. URL : https://asexualagenda.wordpress.com/2019/08/01/lisa-orlandoauthor-of-the-asexual-manifesto-1972/

SIGGY. 2018. «Asexuality in early radical feminism, part 1 » The Asexual Agenda, consulté le 10 mai 2021. URL : https://asexualagenda.wordpress.com/2018/08/29/asexuality-in-early-radical-

feminism-part-1/

\section{NOTES}

1. À ce moment-là, en 1972, The Feminists ne comptait plus que trois membres.

2. AVEN : Asexual Visibility and Education Network [Réseau pour l'Éducation et la Visibilité Asexuelle]. Fondé en 2001 par un activiste ace, David Jay, c'est aujourd'hui le plus gros réseau asexuel du monde. Lien vers la version francophone du forum : https://fr.asexuality.org/

3. Le texte utilise le mot scene. C'est une expression qui renvoie à tout ce qu'il se passe habituellement dans un certain contexte social. Ici, cela fait référence à la drague au bar et (souvent) au coup d'un soir qui suit. 


\section{RÉSUMÉS}

Traduction du document «Asexual Manifesto » de Lisa Orlando. Ce document a été produit dans les milieux féministes voire lesbiens des États-Unis des années 1970. Il a été partagé et diffusé dans les quelques années suivant sa diffusion avant de tomber dans l'oubli jusqu'à une redécouverte en 2019. Il évoque une asexualité qui serait un choix politique visant à contrer le sexisme qui imprègnerait toute relation sexuelle, quels que soient les individus impliqués.

French translation of the Asexual Manifesto by Lisa Orlando. This document was written in the feminists/lesbian circles of the USA in the 1970s. It was published and shared during a few years before falling into oblivion until its rediscovery in 2019. The asexuality it describes is one of a political choice meant to fight against the sexism ingrained in every sexual relationships, no matter who they involve.

\section{INDEX}

Thèmes : Explorations

Keywords : asexuality, radical feminism, sexual orientation, history, US feminism

Mots-clés : asexualité, féminisme radical, orientation sexuelle, histoire, féminisme états-unien

\section{AUTEURS}

\section{ÉLIE GRAU}

Élie Grau est activiste ace et a une formation d'historien. Il a mené un mémoire de master sur le monachisme égyptien, à l'université de Strasbourg. Certifié et actuellement professeur de lettres classiques. 